\title{
Mass Flux in Fire Plumes
}

\author{
E. E. ZUKOSKI \\ California Institute of Technology \\ Pasadena, California 91125
}

\begin{abstract}
A review is given of data that describe the mass flux of gas in large buoyant diffusion flames, with the aim of developing a rational picture for this process as well as a correlation of the data. A brief review of flame-height scaling parameters is followed by a discussion of measurement techniques, the previous work on far-field and fire-plume models, and a description of an effort to develop a rational entrainment model.
\end{abstract}

Keywords: Mass flux, entrainment; diffusion flame, fire plume.

\section{SCALING OF FLAME PHENOMENA}

The appropriate scaling parameters for most of the features of fire plumes have not yet been established with confidence. However, the scaling laws for the size of the diffusion flames have been developed by a number of authors, e.g. Zukoski [1], and some consensus has been reached that a dimensionless heat addition parameter $Q^{*} \equiv \dot{Q} /\left(\rho_{\infty} C_{p \infty} T_{\infty} \sqrt{g D} D^{2}\right)$, or a dimensional parameter that is proportional to $Q^{*}$ such as $\dot{Q} / D^{(5 / 2)}$ can be used as the scaling parameter. Here, $\dot{Q}$ and $D$ are the heat-release rate and fire diameter; $\rho_{\infty}, \mathrm{C}_{\mathrm{p} \infty}$, and $\mathrm{T}_{\infty}$ are the density, specific heat, and temperature of the ambient gas; and $g$ is the acceleration of gravity.

Flame-length data discussed in Zukoski [1] and Hasemi [2] indicate that scaling laws for the visible flame length, $Z_{f}$, and possibly for other parameters of interest including the entrainment rate, have a different form for several ranges of $Q^{*}$ and must be considered separately for each regime. For example, based on flame-length data, three flame-length regimes of interest can be distinguished: $Q^{*} \leq 0.1,0.1 \leq Q^{*} \leq 1.0$, and $1.0 \leq Q^{*}$ and we call these flame regimes 1,2 , and 3. In regime $1, Z_{f} / D$ scales as $\left(Q^{*}\right)^{2}$, the flame length is less than the burner diameter and is proportional to the square of the heat addition per unit area of the burner, and these results appear to be sensitive to the properties of the fuel (Hasemi 1992). In the third regime, $Z_{f} / D$ is larger than 3 and scales as $\left(Q^{*}\right)^{2 / 5}$, and consequently, the flame length is independent of the burner diameter. The second regime is a transition regime between 1 and 3 . Given these 
results, we expect that the plume mass-flux correlation will vary when $Q^{*}$ values move across the regime boundaries defined above. Most accidental fires (see e.g., Hasemi [2] ) fall in the second or transitional regime, and most of the experiments discussed here were carried out in this range even though their size is much less than that required to model exactly full-scale flames.

\section{MASS FLUX MEASUREMENT TECHNIQUES}

Several techniques have been developed for making mass-flux measurements in the fire plume and these are also used in making measurements in adiabatic plumes. Several are discussed here.

Velocity and Temperature Measurements: The first technique involves the measurement of local values of velocity and density at a number of elevations and radial positions in the adiabatic plume or fire plume. These data are then used to obtain time-averaged estimates of the density $\bar{\rho}\{r, z\}$ and velocity $\bar{V}\{r, z\}$ profiles, and the integration of the product over the area of the plume leads to an estimate of the total mass flux in the plume. Use of simultaneously obtained values of the product of $\rho\{r, z\}$ and $V\{r, z\}$ avoids errors caused by turbulent transport. Although making instantaneous values of temperature and velocity in the fire plume is difficult, McCaffrey [3], Cox and Chitty [4and 5], and Weckman and Strong [6] have used variations of this technique to estimate the mass flux as a function of height for fires in the $20-$ to $100-\mathrm{kW}$ range for a few conditions.

Ricou and Spalding Apparatus: Ricou and Spalding [7] developed a second technique that is easily applied to measurements made far from the source of the jet or plume where the velocity in the plume is relatively small, and fluctuation amplitudes are reduced in magnitude and rate. Sensitivity to flame oscillations, such as the puffing phenomena, and the requirement that positions closer to the burner surface than one diameter make application of this technique to fire-plume studies a poor choice. This technique was successfully applied by Ricou and Spalding [7], who made entrainment measurements for the far field of burning jets, momentumcontrolled jets, and buoyant plumes.

Hood Technique: This technique has been applied to making measurements of entrainment rates in adiabatic and fire plumes. The apparatus consists of a large hood that is placed over the fire source to be studied. The hot gas accumulates within the hood and spills under the edges in a layer no more than a few millimeters below the bottom edge of the hood. Mixing processes driven by the fire plume produce a uniform composition of gas within the hood.

The mass flux in the fire plume at the interface is calculated from measurements of the fuelflow rate to the burner and the fuel-air ratio of the gas inside the hood which is determined from a chemical analysis of gas samples withdrawn from the hood after a steady state has been established. The presence of the horizontal interface between hot and ambient gas forces the velocity to remain horizontal near this boundary. This constraint will affect the velocity field of 
the inflowing gas and may cause the entrainment rate at a given height to be a function of the interface height. Thus, the interface may modify the entrainment rate.

The basic premise of this method is the assumption that the interaction between the plume flow and the interface does not produce an additional mass flow across the interface. If we are satisfied to determine the total mass flux across the interface, that produced by the plume and by any interaction, then the technique gives the correct answer, and in most accidental-fire problems this is the quantity of interest. However, if we seek to determine the mass flux in a fire plume rising in a constant-temperature ambient, the technique is open to question because of interactions at the interface; further, the influence of the flat interface on the inflow of ambient gas into the plume may affect the entrainment rate. However, several flowvisualization techniques used to observe the interface indicate that the mass flux due to the interaction is very small.

The entrainment rate into fire plumes was studied with an early version of this technique by $P$. $\mathrm{H}$. Thomas et al. [8] in conjunction with measurements of flow through ceiling vents. This work has been reviewed recently in a more available form by Hinkley [9]. Plumes generated by room-scale fires produced by gas burners and wood cribs were placed beneath a hood-like ceiling with short vertical side walls. The vitiated gas escaped through vents in the ceiling and from under the sides of the containing walls, and the mass flux in the plume at the interface between hot and ambient gas was estimated from observations of the depth of the layer and gas temperatures. Results are presented in terms of a correlation proposed for fires with flames that penetrated the interface, and are given in terms of the perimeter of the fire and distance from the bottom of the burning fuel bed to the interface.

The Caltech group (Zukoski et al. [10], Cetegen et al. [11], Lim [12], Toner [13], and Morehart [14] introduced a hood technique and used hoods that were either $1.2-\mathrm{m}$ or $1.8-\mathrm{m}$ cubes to measure the mass flux as a function of height of the interface in flame and buoyant plumes. Natural gas was the primary fuel but ethylene and propylene flames were also studied by Morehart. The flames were stabilized on 10-, 19-, and 50-cm burners and had heat-release rates of 20 to $100 \mathrm{~kW}$. The interface height was measured from the surface of the burner.

A variation of the hood technique was also used by Beyler [15]. He used a 1.02-m diameter cylindrical hood with a depth of $0.48 \mathrm{~m}$. The bottom of the cylinder was open but the hot gas was withdrawn from a circumferential slot in the side wall to a point $33 \mathrm{~cm}$ above the bottom of the hood. Because the interface in these experiments was typically 10 to $15 \mathrm{~cm}$ thick, compared with the hot-layer depth of less than $30 \mathrm{~cm}$, the definition of the interface height was based on measured temperature or composition gradients. Beyler used this apparatus to measure entrainment rates for a large number of fuels burning in fires stabilized on 13- and 19cm burners.

In experiments carried out by the Caltech group and by Beyler, gaseous fuels were injected through horizontal beds. Beyler also studied pool fires of methanol. The technique used by these investigators allowed a more precise measurement of mass flux than that used by Thomas 
$e t a l .[8]$ but the fire sources used in the large-scale experiments of the latter group are certainly more realistic.

\section{PLUME MODELS}

Adiabatic Plumes: Models for the entrainment into adiabatic and buoyant plumes have typically been based on the work of Morton, Taylor, and Turner [16] and their predictions have been found to be satisfactory for buoyant plumes rising above a point source. The primary problem in applying these results to adiabatic plumes rising above fires is the requirement that the source of the equivalent point-source plume must be located with respect to the level of the fire. Given accurate information concerning the flow at the top of the flame, this location can be calculated; however, because this information is in general not available, correlations must be used.

Experimental data obtained by Cetegen et al. [11] and Zukoski et al. [10] in the hood apparatus described above have been analyzed by Heskestad [16] and Cetegen et al. [11] with the result that several models are now available to predict the elevation of the source for the equivalent point-source plume relative to the fire source. The resulting correlation for the mass flux $\dot{\boldsymbol{m}}_{f}$ for the adiabatic and far-field plume at a point $Z$ above the surface of the burner and $\left(Z+\Delta Z_{f}\right)$ above its effective source located at $\Delta Z_{f}$ below the surface of the burner (see Cetegen et al. 1984), can be written the dimensionless form:

$\dot{\mu}_{f} \equiv \frac{\dot{m}_{f}}{\rho_{\infty} \sqrt{g D D^{2}}}=0.21\left(Q^{*}\right)^{1 / 3}\left(\frac{Z}{D}+\frac{\Delta Z_{f}}{D}\right)^{5 / 3} \quad$ Far field

Fire-Plume Models: Descriptions of the entrainment into the flaming region of the fire, called the fire plume, have not been as satisfactory. Models or correlations have been suggested by a number of investigators including Thomas et al. [8], Steward [, Cetegen et al. [18], McCaffrey [18], Heskestad [17], Hinkley [9], and Delichatsios [20]. The primary results of four of these models or correlations for the prediction of the dependence of the plume mass flux on the height above the fire, $Z$, fire source diameter $D$ or perimeter $\mathbf{P}$, and heat release rate, $Q$, in the flame plume are summarized briefly below:

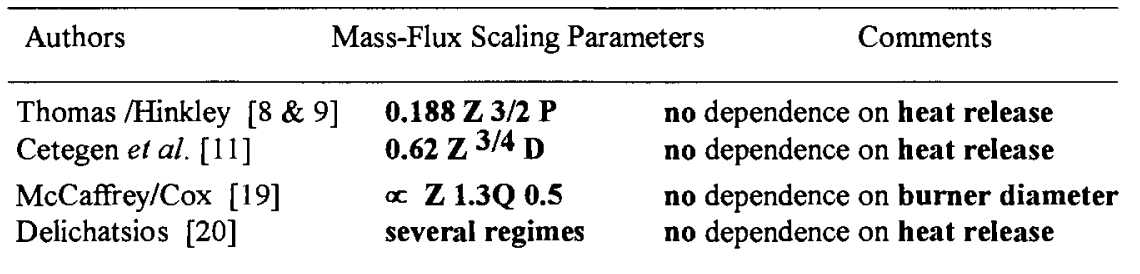




\section{ANALYSIS OF CALTECH EXPERIMENTAL DATA}

Measurements of plume mass-flux rates for a given elevation in the fire plume stabilized on a given burner with a wide range of fuel-flow rates were made by the Caltech group. They found that the plume mass-flux rates were scattered but showed no definite trend with heat-release rates and certainly did not scale linearly or with the square root of the heat-release rate. Entrainment rates obtained by Thomas et al. and Beyler also showed no dependence on heat release rate of the fire.

The mass flux in the plume, obtained by the Caltech group, is shown as a function of the interface height in Figure 1 for a $19-\mathrm{cm}$ burner with heat-release rates from natural-gas flames of about $60 \mathrm{~kW}$ and with $Q^{*}=4.5$. The center arrow indicates the time-averaged flame height; the other arrows indicate the upper and lower bounds for the flame fluctuation. Both the far-field and near-field correlations proposed here are shown as solid lines. The far-field data are fit accurately by the correlation discussed above. The origin of this curve is offset from the elevation of the plane of the burner by an amount given by a correlation suggested by Cetegen et al.[11]. The near-field data are fit by a straight line.

This type of behavior was found for the flames studied here that had $0.1 \leq Q^{*} \leq 17$, although the data for most conditions are sparse in the flame-plume region. These data suggest that mass flux for both the near and far fields can be expressed by single correlations which intersect near the top of the flame. Data and correlations for the 19-cm burner with three heat-release rates are shown in Figure 2, at a larger scale for the near-field region alone. In this figure, the correlation of Delichatsios [19] appears as the upper dashed curve, and that from Thomas et al, [8] as the lower curve with shorter dashes. The open circles are the data of Cetegen, and the squares are the data of Toner and Morehart. In the flame region, the data are nicely fit by a simple straight line which agrees with a correlation first presented by Heskestad [17]. Neither Delichatsios' nor Thomas' correlation agrees well with the fire-plume data.

A second collection of data of this type for the fire plume are shown in Figure 4 for data obtained with a $50-\mathrm{cm}$ diameter burner with natural gas as the fuel. These data and much of the data of Beyler again suggest that the mass flux varies roughly linearly with height.

The Caltech data for the flame or near field region can be most simply correlated by the expression $\dot{m}_{n}=C_{e f} Z D$, for the whole range of values for $Q^{*}$. The parameter $C_{e f}$, defined

as $\left(\dot{m}_{n} / Z D\right)$, does depends on $Q^{*}$ and the dependence is shown in Figure 3. The data indicate that, when $0.1 \leq Q^{*} \leq 4, C_{e f}$ is about 0.62 , and is independent of the heat addition rate and $Q^{*}$. The range over which $C_{e f}$ is constant coincides almost perfectly with the second flame regime and also with the range of $Q^{*}$ that is the most interesting for application to accidental fires. 
When $Q^{*} \geq 4.0$, the value of $C_{e f}$ increases strongly with $Q^{*}$. Unfortunately, this change is also associated with a change in burner diameter and the available data do not give a clear indication that the data for the 10-cm burner would join the curve for the 19- and 50-cm burner data at the point where the two curves intersect. Thus, we can not say definitely that the change observed around $Q^{*}=4.0$ is due to the expected change as $Q^{*}$ increases from the second to the third flame regime or to a change associated with the different burner size. An example of the latter explanation is that the flames on the $10-\mathrm{cm}$ burner, at the low fuel-flow rates required to produce a $Q^{*}$ value near 4.0 , appear much more laminar than do the larger fires on the larger burners. However, at much higher flow rates, and consequently higher values for $Q^{*}$, the flames stabilized on the 10 -cm burner appear to be more turbulent but still have much higher values for $C_{e f}$. Finally, the single data point near a $Q^{*}$ value of 0.1 suggests that $C_{e f}$ may decrease as the flow enters the first flame regime. Clearly, more data obtained with larger-diameter burners is required to clarify these transitions.

The plume mass-flux data from Beyler for flames stabilized on 13- and 19-cm burners but with a different type of hood agree with some of the characteristics of the data collected by the Caltech group, i.e. no dependence on heat-release rate and a linear dependence on height above the burner. However, the Caltech data are generally up to twice as large.

\section{DISCUSSION OF A CORRELATION FOR $0.1 \leq Q^{*} \leq 4$}

The major problem with the mass-flux correlation discussed above for the fire plume in the intermediate regime, $0.1 \leq Q^{*} \leq 4.0$, is that it is not dimensionally correct. Some other correlation must be sought; the clue is given by the fact that in Figures 2 and 4 the curves of Thomas' correlation are almost parallel to the upper part of the experimental data. This suggests that the experimental results can be correlated by an equation of the form suggested by Thomas [8], $\dot{m}_{n}=0.59 Z^{3 / 2} D$, that is rewritten for the Caltech data with the origin for the $Z$ axis located at $\Delta Z_{n}$ below the fire surface. That is,

$$
\dot{\mu}_{n} \equiv \frac{\dot{m}_{n}}{\rho_{\infty} \sqrt{g D} D^{2}}=0.18\left(\frac{Z}{D}+\frac{\Delta Z_{n}}{D}\right)^{3 / 2} \quad \text { Fire Plume with } 0.1<Q^{*}<4.0
$$

with the effective near-field plume height written as $Z_{n f} \equiv\left(Z+\Delta Z_{n}\right)$. Addition of the constants and $\rho_{c o}$ does not change the nature of the correlation since they were unchanged during the experiments. The value of the product of these constants, $\left(C_{T} \rho_{\infty} \sqrt{g} \pi\right)$, is about 0.62 in $m k s$ units for the Caltech data and thus is very close to the value 0.59 that was suggested much earlier by Thomas. Thomas also suggested that the perimeter of the fire, not 
the diameter, was the correct scaling parameter in this regime and this distinction will be important when fuel sources other than circular burners are considered.

The use of an offset is not as arbitrary as may appear here since many of Thomas' experiments were conducted with wood cribs placed on the laboratory floor and the height to the interface was measured from the floor. Direct comparison between these fires with a vertically distributed fuel source and bumer flames, in which the flame is stabilized on a horizontal plane surface, is difficult and this difference in fuel geometry suggests that an offset of some type might be required to correlate the data.

This correlation is compared with the 50-cm burner data in Figure 4 and the data show that the use of the offset allows a good representation of the experimental results. An equally

satisfactory correlation was obtained for the 19-cm data. The required offset $\Delta Z_{n}$ is about 12 and $18 \mathrm{~cm}$ for the $50-$ and $19-\mathrm{cm}$ burners, that is, it is between one quarter and one burner diameter, and the limited data suggest that it is insensitive to changes in the heat-addition rate.

The correlation proposed here can be rationalized by the following argument: using the derivation suggested by Morton et al. for their plume analysis, assume that the entrained mass flux is proportional to the ambient density $\rho_{\infty}$, the perimeter of the fire $\pi D$, and the velocity of the flow $V$ at height $Z$; i.e., that

$d\left(\dot{m}_{n}\right)=\rho_{\infty}(\pi D) V d Z$

We make the rather arbitrary assumption that the flow speed $\mathrm{V}$ is proportional to $\sqrt{g Z}$ since

$V \propto \sqrt{\left(\frac{\rho_{\infty}-\rho_{\text {flame }}}{\rho_{\text {flame }}}\right) g Z} \approx \sqrt{\left(\frac{T_{\text {flame }}-T_{\infty}}{T_{\infty}}\right) g Z} \propto \sqrt{g Z}$

when the density and temperature of the gas are approximately constant in the fire plume. Integration over the height of the product then gives equation (2) suggested above. The proposed correlation appears to represent the data satisfactorily and can be rationalized even though the effects of the puffing have been ignored.

The calculation scheme for the mass flux in the plume proposed here is that equation (1) should be used for the fire plume and equation (2) for the far-field plume. This logically requires that the two curves should cross at some position near the top of the flame and the data for the experiments discussed here do follow that pattern, e.g., Figure 1. Because the mass flux in the near field or fire plume scales with $\left(Z_{n f} / D\right)^{3 / 2}$ and in the far field as $\left(Z_{f f} / D\right)^{5 / 3}$, the intersection will be acute for values of flame length near one diameter.

Thus, the location and nature of this intersection depends strongly on the far- and near-field offsets $\Delta Z_{f}$ and $\Delta Z_{n}$ and this dependence becomes more important as the flame height decreases. Although correlations exist for $\Delta Z_{f}$, data are not yet available which will allow $\Delta Z_{n}$ to be determined accurately. The offsets are important near the intersection because the 
flame lengths in regime 2 vary between one and three burner diameters in height and consequently offset values that place the origin for the curves at positions from $(0.5 \mathrm{D})$ below to $(I . O D)$ above the burner surface can make a large difference in height of the intersection.

The scaling proposed in equations (1) and (2) has the property that for given values of $(Z / D)$ and $Q^{*}$, the two mass-flow rates scale in an identical manner with the diameter and heatrelease rate if the offsets also scale with the diameter and, at most, a function of $Q^{*}$. Under these circumstances, the value for $Z / D$ at the intersection of the two curves will not change with burner diameter or heat release rate when the value of $Q^{*}$ is fixed.

The application of the model depends on obtaining accurate representations for both offsets. Data for the fire-plume offset is available for only two burner diameters. The offsets obtained from the limited data presently available suggest that the offset is proportional to the burner diameter; the data are not sufficient to define accurately the dependence of the offset on the parameters of the system. For larger values of $Q^{*}$, the offset for the far-field entrainment rate is negative, that is, the origin lies above $Z=0$, and the intersection of the curves is more obtuse.

Although experimental entrainment data for large fires are required to clarify these modeling results, the proposed correlations do give an accurate description of the experimental data for fires up to one-hundred kilowatts. The scaling proposed here depends on the parameter $Q^{*}$ and the assumption that the offsets scale with burner diameter $D$ and some function of $Q^{*}$. If these assumptions are correct, and if values for the offset for the fire-plume data can be obtained, this scaling procedure will give a good representation of the plume mass flux in both the fire plume and the far-field plume.

\section{ACKNOWLEDGMENT}

This work was supported in part by a grant from the Building and Fire Research Institute, and the National Institute of Science and Technology under the guidance of Dr. L. Cooper. 


\section{REFERENCES}

1. Zukoski, E. E., "Fluid Dynamic Aspects of Room Fires," Proc. of First International Symp. Fire Safety Science, p. 1, (1986)

2. Hasemi, Y., "Deterministic Properties of Turbulent Diffusion Flames from Low $Q^{*}$ Fires, "Fire Sci. and Tech., 7, No. 2, p.27--34, (1987)

3. McCaffrey, B. J., "Purely Buoyant Diffusion Flames, Some Experimental Results," NBSIR 79-1910, Nat. Bur. Stand., Wash. D. C., (1979)

4. Cox, G. and Chitty, R., "A Study of the Deterministic Properties of Unconfined Fire Plumes," Comb. and Flame 39: 191, (1980)

5. Cox, G. and Chitty, R., "Some Stochastic Properties of Fire Plumes," Fire and Materials 6: 127 (1982)

6. Weckman, E. J. and Strong, A. B., NIST Conference on Fire Research, (1992)

7. Ricou, F. P. and Spalding, B. P., "Measurements of Entrainment by Axisymmetric Jets," J. Fluid Mechanics, p. 11, (1961)

8. Thomas, P. H. et al, "Investigation into the Flow of Hot Gas in Roof Venting," Technical Paper No. 7, HMSO, London, (1963)

9. Hinkley, P. L., "Rate of "Production" of Hot Gas in Roof Venting Experiments," Fire Safety Journal, 10: 57, (1986)

10. Zukoski, E. E., Kubota, T. and Cetegen, B., "Entrainment in Fire Plumes," Fire Safety Journal, 3: 107, (1980-81)

11. Cetegen, B. M., Zukoski, E. E., and Kubota, T., "Entrainment in the Near and Far Fields of Fire Plumes," Comb. Sci. and Tech., 39: 305, (1984)

12. Lim, C., I. Mixing in Doorway Flows, II. Entrainment in Fire Plumes, Engineer's Degree Thesis, Calif. Institute of Tech., June (1984)

13. Toner, S. J., Entrainment, Chemistry, and Structures of Fire Plumes, Ph.D. Thesis, Calif. Institute of Tech., Pasadena, Calif. (1987)

14. Morehart, J. H. Ph.D., "Species Production in Fires Burning in Two-Layered and Honogeneously Vitiated Environmernts," Thesis, Calif. Institute of Tech., Pasadena, Calif. (1991)

15. Beyler, C. L. ,"Development and Burning of a Layer of Products of Incomplete Combustion Generated by a Buoyant Diffusion Flame", Thesis, Harvard Uiversity, Boston, Mass. (1983).

16. Morton, B. R., Taylor, G. I., and Turner, J. S., " Turbulent Gravitational Convection from Maintained Instantaneous Sources,", Proc. Royal Soc, A 234: p. 1, (1956)

17. Heskestad, G., "Fire Plume Entrainment According to Two Competing Assumptions," $21^{\text {St }}$ Comb. (Int.) Symp, p. 111-120, (1985)

18. Steward, F, R., "Determination of the Height of Diffusion Turbulent Flames," Comb. Sci. and Tech., 2: 203, (1970)

19. McCaffrey, B. and Cox, G., NBSIR 82-2473., Nat. Bur. Stand., Wash. D. C. (1982)

20. Delichatsios, M. A., "Air Entrainment into Buoyant Jet Flames and Pool Fires," Comb. and Flame, 70: 33-46, (1987) 


\section{FIGURES}

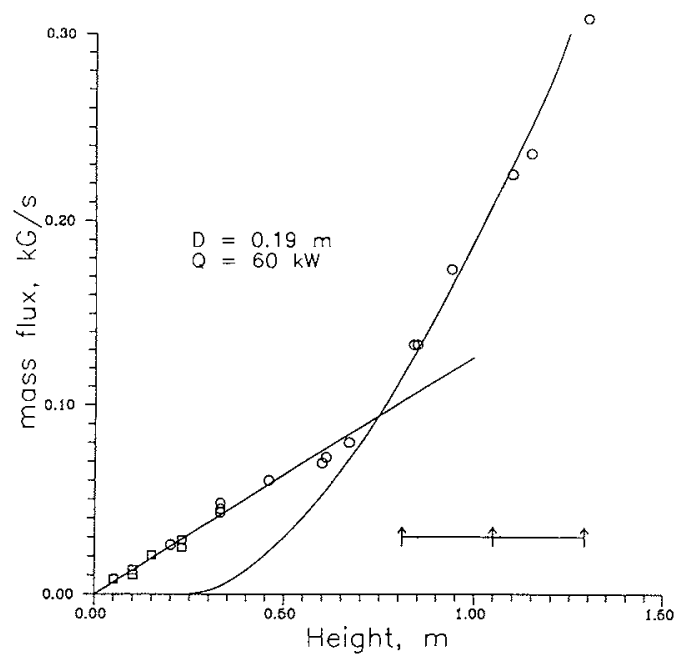

Figure 1. Plume Mass Flux versus Height for 60-kW Natural-Gas Flames on a 19-cm Diameter Burner.

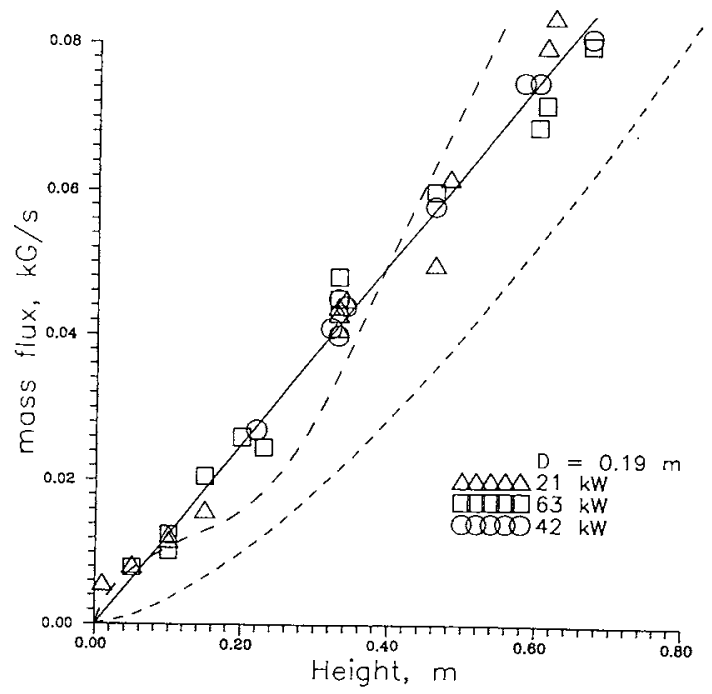

Figure 2. Plume Mass Flux versus Height for Natural-Gas Flames on a 19-cm Burner. 


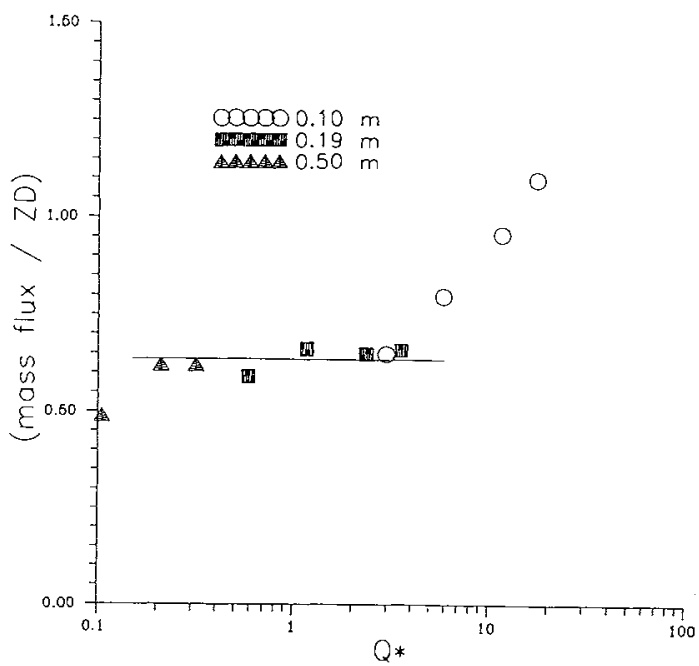

Figure 3. Plume Mass Flux Normalized by $\mathrm{ZD}$ versus $\mathrm{Q}^{*}$ for Three Burners.

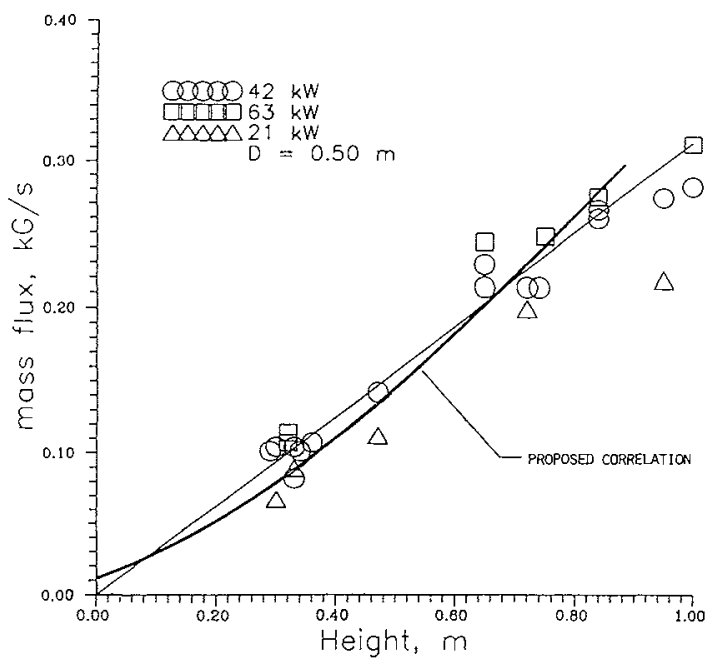

Figure 4. Comparison of Plume Mass Flux for the 50-cm Burner with the Proposed Correlation. 
